\title{
Optimal Control Strategy for a Fully Determined HIV Model
}

\author{
Mohammad Shirazian ${ }^{1}$, Mohammad Hadi Farahi ${ }^{1,2}$ \\ ${ }^{1}$ Department of Applied Mathematics, Ferdowsi University of Mashhad, Mashhad, Iran \\ ${ }^{2}$ The Center of Excellence in Modeling and Computations in Linear and Nonlinear Systems \\ Ferdowsi University of Mashhad, Mashhad, Iran \\ E-mail:mo.shirazian@stu-mail.um.ac.ir,farahi@math.um.ac.ir \\ Received January 25, 2010; revised March 21, 2010; accepted June 22, 2010
}

\begin{abstract}
This paper shows how mathematical methods can be implemented to formulate guidelines for clinical testing and monitoring of HIV/AIDS disease. First, a mathematical model for HIV infection is presented which the measurement of the $\mathrm{CD}^{+}{ }^{T}$ cells and the viral load counts are needed to estimate all its parameters. Next, through an analysis of model properties, the minimal number of measurement samples is obtained. In the sequel, the effect of Reverse Transcriptase enzyme Inhibitor (RTI) on HIV progression is demonstrated by using a control function. Also the total cost of treatment by this kind of drugs has been minimized. The numerical results are obtained by a numerical method in discretization issue, called AVK.
\end{abstract}

Keywords: HIV/AIDS, Mathematical Modeling, System Identification, Control Theory, Immunotherapy

\section{Introduction}

Despite tremendous effort for mathematical modeling of HIV/AIDS (for example, see [1-4]), estimation of model parameters has not been attended a lot. For example, in $[2,5,6]$, only the virus clearance rate and the death rate of infected $\mathrm{CD}^{+} \mathrm{T}$ cells have been estimated. The importance of parameter estimation in models, is due to predicting "set-points" in the early infection stage for making the desired treatment decisions (See [7]).

One of the objectives of this paper is presenting a realistic model, i.e. the basic model of HIV, and estimating all its parameters. It is necessary to mention that one can identify all of the model parameters by using measured output (For more details see [4]).

Another objective is to add a control function to the identified basic model which plays the role of reverse transcriptase enzyme inhibitor drug in disease progression.

In the sequel, the optimal control model of HIV will be solved by a method in discretization issue, called AVK.

Numerical results are obtained using mathematical softwares, LINGO and MATLAB.

\section{Translating Biological Knowledge to Ordinary Differential Equations (ODE)}

To make ODE's from biological knowledge, first we need some syntax. For example, if we denote the count of uninfected and infected $\mathrm{CD} 4^{+} \mathrm{T}$ helper cells, with $a$ and $b$, respectively, the syntax " $a \rightarrow 0$ " can be used to present this biological descriptions: "Uninfected $\mathrm{CD} 4{ }^{+} \mathrm{T}$ cells die" and the syntax " $a+b \rightarrow b+b$ " can present: "The reaction between two infected and uninfected $\mathrm{CD} 4^{+} \mathrm{T}$ cells produces two infected $\mathrm{CD} 4^{+} \mathrm{T}$ cells". Now, for translating these syntaxes to the corresponding ODE's, we use Mass action law. This law says: "The rate of change of products is proportional to the product of reactants concentration". So if the syntax " $a+b \rightarrow c$ " is obtained, according to the mass action law, we can write $\dot{c}=k a b$, for $k>0$, where $\frac{d c}{d t}$ is denoted by $\dot{c}$. Two other reactions in the previous syntax is dying $a$ and $b$ reactants, while producing $c$. So we have also these two ODE's as: $\dot{a}=-k a b$ and $\dot{b}=-k a b$, for $k>0$. Finally, the desired ODE, corresponding to the syntax " $a+b \rightarrow c$ " is 


$$
\begin{aligned}
& \dot{c}=k a b, \\
& \dot{a}=-k a b, \\
& \dot{b}=-k a b .
\end{aligned}
$$

Obviously, the rate of change of a product is the sum of changes from all reactions.

\section{HIV Basic Model}

The target cells of HIV infection are lymphocyte helper cells, specially $\mathrm{CD}^{+}{ }^{+} \mathrm{T}$ cells. These cells become infected and begin to produce free virions. The main fact about $\mathrm{HIV}$ infection, is reducing the count of $\mathrm{CD}^{+} \mathrm{T}$ cells, which have an essential role in protecting body against different pathogens. So it is important to understand the dynamics of $\mathrm{CD}^{+} \mathrm{T}$ cell count as a function of time. In HIV infection basic model, three groups of molecules are considered; Uninfected CD ${ }^{+} \mathrm{T}$ cells (T), infected $\mathrm{CD}^{+} \mathrm{T}$ cells (I) and viral load (V). Biological descriptions, translation to reactions and corresponding ODE's are presented in Table 1.

Now, according to Table 1 and Section 2, the complete ODE model, which is sum of contributions from all reactions, is as follows:

$$
\begin{aligned}
& \dot{T}=s-d T-\beta T V, \\
& \dot{I}=\beta T V-\mu I, \\
& \dot{V}=k I-c V .
\end{aligned}
$$

\section{Properties of HIV Basic Model}

There are two advantages to show the virous propagation in HIV disease, by the basic model (1).

1) From medical point of view, one important subject is the relative steady viral level during the asymptomatic stage of an HIV infection. This level is called "set-point". When body reaches this level, immune system develops HIV antibodies and begins to attempt to fight the virus. The higher the viral load of the set point, the faster the virus will progress to full blown AIDS (See [8]).

It can be shown that set-point is the amount of $\mathrm{V}$, in the equilibrium of virus depicted by the model (1), that is

$$
V^{*}=\frac{k s}{\mu c}-\frac{d}{\beta} .
$$

2) It can be seen that a model of such a simple nature is able to adequately reflect the disease progression from the initial infection to an asymptomatic stage after the set-point is reached (See [9]).

\section{Estimation of Models Parameters Using Discretization}

In this section, our aim is to estimate all parameters of HIV basic model (1). Clinically all six variables in model (1), can be measured. Since the cost of quantifying the infected cells is much higher, we are going to omit variable I, initially. For this, let $y_{1}=T$ and $y_{2}=V$. After some calculations, model (1) can be changed to:

$$
\begin{aligned}
& \dot{y}_{1}=\alpha_{1}+\alpha_{2} y_{1}+\alpha_{3} y_{1} y_{2} \\
& \ddot{y}_{2}=\alpha_{4} \dot{y}_{2}+\alpha_{5} y_{2}+\alpha_{6} y_{1} y_{2}
\end{aligned}
$$

where

\begin{tabular}{|c|c|c|c|}
\hline Biological description & Translation to reactions & Reaction rate & Translation to ODE \\
\hline $\mathrm{CD} 4^{+} \mathrm{T}$ cells production & $0 \rightarrow T$ & $s$ & $\dot{T}=s$ \\
\hline $\mathrm{CD}^{+} \mathrm{T}$ cells natural death & $T \rightarrow 0$ & $d$ & $\dot{T}=-d T$ \\
\hline $\mathrm{CD} 4{ }^{+} \mathrm{T}$ cells become infected by virus & $T+V \rightarrow I+V$ & $\beta$ & $\begin{array}{l}\dot{T}=-\beta T V \\
\dot{I}=\beta T V\end{array}$ \\
\hline Infected $\mathrm{CD} 4^{+} \mathrm{T}$ cells death & $I \rightarrow 0$ & $\mu$ & $\dot{I}=-\mu I$ \\
\hline Virus replication in infected $\mathrm{CD} 4^{+} \mathrm{T}$ cells & $I \rightarrow I+V$ & $k$ & $\dot{V}=k I$ \\
\hline Virus natural death & $V \rightarrow 0$ & $c$ & $\dot{V}=-c V$ \\
\hline
\end{tabular}

$$
\alpha=\left[\begin{array}{l}
\alpha_{1} \\
\alpha_{2} \\
\alpha_{3} \\
\alpha_{4} \\
\alpha_{5} \\
\alpha_{6}
\end{array}\right]=\left[\begin{array}{c}
s \\
-d \\
-\beta \\
-\mu-c \\
-\mu c \\
k \beta
\end{array}\right] .
$$

The vector $\alpha$ defines a one-to-one map for $\beta \neq 0$ and $\mu \neq c$. Therefore the identification of the original parameters of (1) is equivalent to the identification of $\alpha$. It is known that for most HIV patients, $\beta \neq 0$ and $\mu<c$ (See [7]). In this case, the following inverse map can be defined:

Table 1. HIV basic model interactions. 


$$
\left[\begin{array}{l}
s \\
d \\
\beta \\
\mu \\
c \\
k
\end{array}\right]=\left[\begin{array}{c}
-\alpha_{1} \\
-\alpha_{2} \\
-\alpha_{3} \\
\frac{-\alpha_{4}-\sqrt{\alpha_{4}{ }^{2}+4 \alpha_{5}}}{2} \\
\frac{-\alpha_{4}+\sqrt{\alpha_{4}{ }^{2}+4 \alpha_{5}}}{2} \\
-\frac{\alpha_{6}}{\alpha_{3}}
\end{array}\right] .
$$

Since there are three unknown parameters in each of Equation (2) and (3), it is necessary to generate at least two other equations based on each of them. This will be achieved by differentiating (2) and (3) more times, and produce upper derivatives of $y_{1}$ and $y_{2}$. So one can concludes that at least four measurements of $y_{1}, \mathrm{CD}^{+} \mathrm{T}$ cell count, and five measurements of $y_{2}$, viral load, are needed for a complete determination of model (1) parameters (See [7]).

Assume that the following measurements are available.

By discretization of Equations (2) and (3), and substituting the approximated values of first derivative of $y_{1}$ and the first and second derivatives of $y_{2}$, we found that

$$
\begin{aligned}
& \alpha_{1}+y_{1}{ }^{i} \alpha_{2}+y_{1}{ }^{i} y_{2}{ }^{i} \alpha_{3}=\frac{y_{1}^{i+1}-y_{1}{ }^{i}}{d_{i+1}}, i=0,1,2 \\
& \frac{y_{2}^{i+1}-y_{2}{ }^{i}}{d_{i+1}} \alpha_{4}+y_{2}{ }^{i} \alpha_{5}+y_{1}{ }^{i} y_{2}{ }^{i} \alpha_{6}= \\
& \quad \frac{1}{d_{i+1}}\left(\frac{y_{2}{ }^{i+2}-y_{2}{ }^{i+1}}{d_{i+2}}-\frac{y_{2}{ }^{i+1}-y_{2}{ }^{i}}{d_{i+1}}\right), i=0,1,2
\end{aligned}
$$

Or in matrix form, we have

$$
\left[\begin{array}{lll}
1 & y_{1}{ }^{0} & y_{1}{ }^{0} y_{2}{ }^{0} \\
1 & y_{1}{ }^{1} & y_{1}{ }^{1} y_{2}{ }^{1} \\
1 & y_{1}{ }^{2} & y_{1}{ }^{2} y_{2}{ }^{2}
\end{array}\right]\left[\begin{array}{l}
\alpha_{1} \\
\alpha_{2} \\
\alpha_{3}
\end{array}\right]=\left[\begin{array}{c}
\frac{y_{1}{ }^{1}-y_{1}{ }^{0}}{d_{1}} \\
\frac{y_{1}{ }^{2}-y_{1}{ }^{1}}{d_{2}} \\
\frac{y_{1}{ }^{3}-y_{1}{ }^{2}}{d_{3}}
\end{array}\right]
$$

Similar matrix form can be obtained from (6). Thus, the variables $\alpha_{i}, i=1,2, \ldots, 6$ and then from (4), all of the basic model parameters can be calculated. As an example, we considered the basic model (1), where the following estimated parameters are as $\mathrm{Xia}$ [7].

$$
\begin{aligned}
& s=7, d=0.007, \quad \beta=0.00000042163, \\
& \mu=0.0999, \quad c=0.2, \quad k=90.67 .
\end{aligned}
$$

Table 2. Available measurements for the count of $\mathrm{CD4}^{+} \mathrm{T}$ cells and viral load.

\begin{tabular}{ccc}
\hline Time $(t)$ & CD4 $^{+}$T cell count $\left(y_{1}\right)$ & Viral load $\left(y_{2}\right)$ \\
\hline$t_{0}$ & $y_{1}{ }^{0}$ & $y_{2}{ }^{0}$ \\
$t_{0}+d_{1}$ & $y_{1}{ }^{1}$ & $y_{2}{ }^{1}$ \\
$t_{0}+d_{1}+d_{2}$ & $y_{1}{ }^{2}$ & $y_{2}{ }^{2}$ \\
$t_{0}+d_{1}+d_{2}+d_{3}$ & $y_{1}{ }^{3}$ & $y_{2}{ }^{3}$ \\
$t_{0}+d_{1}+d_{2}+d_{3}+d_{4}$ & - & $y_{2}{ }^{4}$ \\
\hline
\end{tabular}

The solution of model (1) for $t \in[0,1000]$, with the initial values $T_{0}=1000, I_{0}=0$ and $V_{0}=7000$, can be determined using the well-known numerical methods like RK4. The graphs of the propagation of healthy $\mathrm{CD} 4{ }^{+} \mathrm{T}$ cells, infected $\mathrm{CD} 4^{+} \mathrm{T}$ cells and virous loads, respectively, are shown in Figure 1.

\section{HIV Infection Optimal Control Model}

There are three convenient groups of drugs for AIDS retroviral therapy; Reverse transcriptase, Protease, and Integrase enzyme inhibitors. In this section, we study the role of reverse transcriptase inhibitors. The main action of this kind of drugs is preventing uninfected lymphocyte cells, to be infected by viral load. According to Table 1,
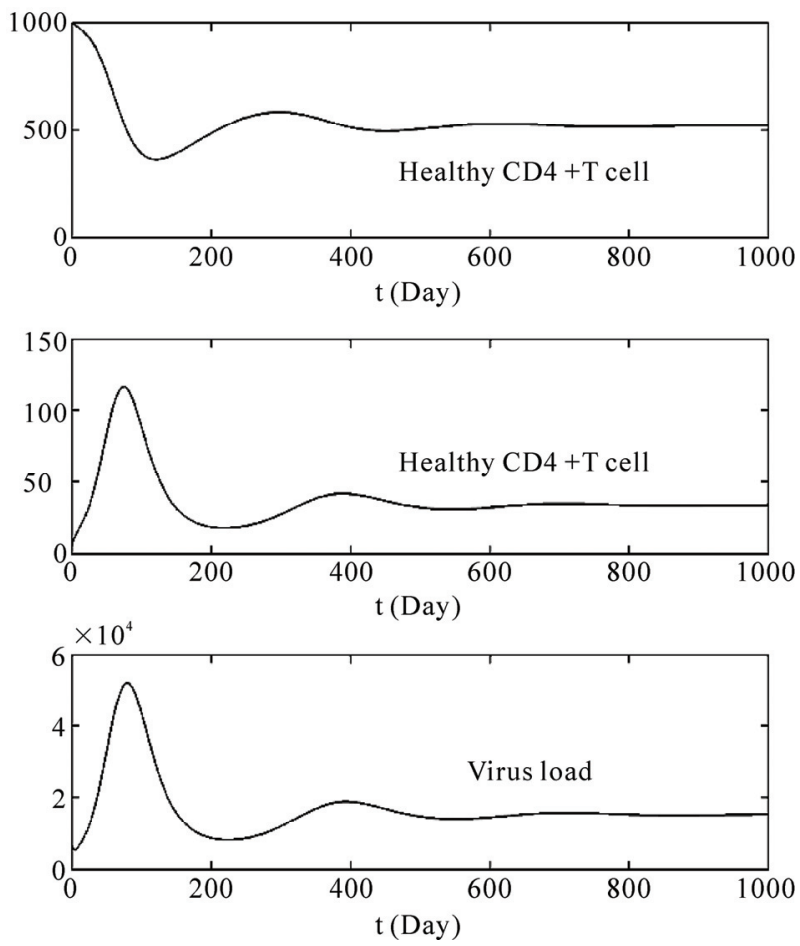

Figure 1. The solution of basic model of HIV, model (1). 
this action is equivalent to the reaction $T+V \rightarrow I+V$. So we control the first equation to prevent the transmission of uninfected cells to infected ones. This control function is called $u(t)$, where $0 \leq u(t) \leq 1$. The most drug efficiency is in the case $u \equiv 1$ which means $\mathrm{CD}^{+} \mathrm{T}$ cells are not infected by viral load anymore. At the other side, $u \equiv 0$ is the case which the drug does not change the disease progression. By above argument, the control system is as:

$$
\begin{aligned}
& \dot{T}=s-d T-\beta T V(1-u), \\
& \dot{I}=\beta T V(1-u)-\mu I, \\
& \dot{V}=k I-c V .
\end{aligned}
$$

Using [10], consider the objective functional to be defined as:

$$
J(T, u)=\int_{t_{0}}^{t_{f}}\left[T(t)-\frac{1}{2} \alpha u(t)^{2}\right] d t
$$

where $\alpha=110$. Our goal is maximizing the objective functional (9) subject to the control system (8); that is, maximizing the total count of $\mathrm{CD}^{+} \mathrm{T}$ cells and minimizing the costs of treatment by applying some RTI drugs.

The solution of this optimal control problem should be calculated by numerical methods. We have used a special discretization method, called AVK.

For a detailed explanation of this method, see [11]. lem,

In AVK method, for solving the optimal control prob-

$$
\operatorname{Min} J(x, u)=\int_{t_{0}}^{t_{f}} g(x(t), u(t), t) d t
$$

Subject to:

$$
\begin{aligned}
& \dot{x}(t)=f(x(t), u(t), t), \quad t \in\left[t_{0}, t_{f}\right] \\
& x\left(t_{0}\right)=x_{0}, \quad x\left(t_{f}\right)=x_{f}
\end{aligned}
$$

the following steps should be applied:

Step 1. Form the total error function $E_{1}$ as:

$$
E_{1}(x, u)=\int_{t_{0}}^{t_{f}}\|\dot{x}(t)-f(x(t), u(t), t)\| d t
$$

Step 2. Combine the total error function with the objective functional (10) as follows:

$$
\begin{aligned}
\operatorname{Min} \int_{t_{0}}^{t_{f}} & \left\{\lambda_{1} g(x(t), u(t), t)\right. \\
+ & \left.\lambda_{2}\|\dot{x}(t)-f(x(t), u(t), t)\|\right\} d t
\end{aligned}
$$

$$
\text { subject to: } x\left(t_{0}\right)=x_{0}, x\left(t_{f}\right)=x_{f}
$$

where nonnegative numbers $\lambda_{1}$ and $\lambda_{2}$ are two given weights and $\lambda_{1}+\lambda_{2}=1$.

Step 3. In order to control the error, add the following constraint,

$$
E_{1}(x, u) \leq \epsilon
$$

to the optimal control problem in Step 2. So the modified optimal control problem (10)-(11) can be formulated as:

$$
\begin{aligned}
\operatorname{Min} \int_{t_{0}}^{t_{f}}\{ & \lambda_{1} g(x(t), u(t), t) \\
& \left.+\lambda_{2}\|\dot{x}(t)-f(x(t), u(t), t)\|\right\} d t
\end{aligned}
$$

subject to :

$$
\begin{gathered}
\int_{t_{0}}^{t_{f}}\|\dot{x}(t)-f(x(t), u(t), t)\| d t \leq \epsilon \\
x\left(t_{0}\right)=x_{0}, \quad x\left(t_{f}\right)=x_{f}
\end{gathered}
$$

Step 4. Calculate $u\left(t_{i}\right)$ by minimizing the optimal control problem (13) using discretization method.

For example, if the norm function $\|\cdot\|$, is norm 1 , then one can solve the following optimization problem:

$$
\begin{gathered}
\operatorname{Min} h \sum_{h=0}^{n-1}\left\{\lambda_{1} g\left(x_{i}, u_{i}, t_{i}\right)+\lambda_{2}\left\|\dot{x}_{i}-f\left(x_{i}, u_{i}, t_{i}\right)\right\|_{1}\right\} \\
\text { subject to }: h \sum_{h=0}^{n-1}\left\|\dot{x}_{i}-f\left(x_{i}, u_{i}, t_{i}\right)\right\|_{1} \leq \epsilon \\
x\left(t_{0}\right)=x_{0}, \quad x\left(t_{f}\right)=x_{f}
\end{gathered}
$$

where $h=\frac{t_{f}-t_{0}}{n}, t_{i}=t_{0}+i h, x_{i}=x\left(t_{i}\right), \quad u_{i}=u\left(t_{i}\right)$ and $\dot{x}_{i}=\dot{x}\left(t_{i}\right) \approx \frac{x_{i+1}-x_{i}}{h}$ for $i=0,1, ., n-1$ and $n \in \mathbb{N}$.

Step 5. By the means of $u\left(t_{i}\right)$ for every $t_{i}$, from (11), it is easy to find $x\left(t_{i}\right)$, for any $i, i=0,1, ., n-1$.

We use this technique to solve the control problem (8) with the objective functional (9). The parameters used in the basic control model (8) are exactly as (7). Assume that the treatment begins when $\mathrm{CD} 4{ }^{+} \mathrm{T}$ cells reach their minimum count, in the absence of drug.

According to Figure 1, $T(129)=363$ is the minimum count of $\mathrm{CD}^{+} \mathrm{T}$ cells. So the treatment interval is $[129,1000]$ day. Also, note that by Figure 1, at $\mathrm{t}=129$, we have $I(129)=57$ and $V(129)=28860$.

Now, we divide $[129,1000]$ into $n$ parts with length $h$. The discretization form of (14) is:

$$
\begin{aligned}
\operatorname{Max} & h \sum_{h=0}^{n-1}\left\{\lambda_{1}\left[T_{i}-\frac{1}{2} \alpha u_{i}^{2}\right]\right. \\
& -\lambda_{2}\left[\left|\dot{T}_{i}-\left(s-d T_{i}-\beta T_{i} V_{i}\left(1-u_{i}\right)\right)\right|\right. \\
& +\left|\dot{I}_{i}-\left(\beta T_{i} V_{i}\left(1-u_{i}\right)-\mu I_{i}\right)\right| \\
& \left.\left.+\left|\dot{V}_{i}-\left(k I_{i}-c V_{i}\right)\right|\right]\right\}
\end{aligned}
$$

subject to: $T_{i}, I_{i}, V_{i} \geq 0, \quad 0 \leq u_{i} \leq 1, \quad \forall i=0,1,2, \ldots, n$

$$
T_{0}=363, I_{0}=57, V_{0}=28860
$$



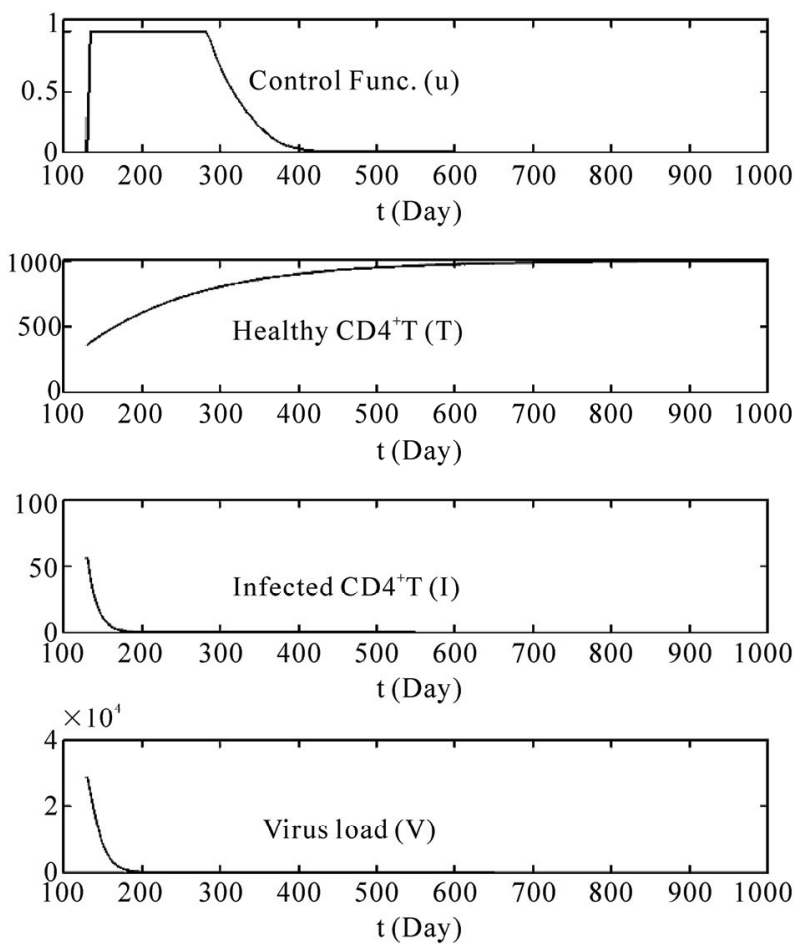

Figure 1. The solution of optimal control problem (8)-(9), using AVK method.

where assumed $\lambda_{1}=\lambda_{2}=\frac{1}{2}$.

The results of this optimization problem which obtained by LINGO and MATLAB softwares for $n=200$ and $\epsilon=10^{-6}$, are depicted in Figure 2.

\section{Conclusions}

In this paper, the parameter of the basic model of HIV/ AIDS is estimated only by measurement of the $\mathrm{CD}^{+} \mathrm{T}$ cells and the viral load count. Since the suggested models for HIV, or infectious diseases like consumption, cholera, influenza and etc., have unknown parameters which should be estimated, one can use the proposed method in this paper to estimate the parameters of such models.

One of the most important kinds of drug treatments for
HIV immunotherapy is assumed. One can investigate the effects of other drugs, like Protease enzyme inhibitors in preventing AIDS progression. In these cases, one can use the described discretization method for solving such optimal control problems.

\section{References}

[1] D. Covert and D. Kirschner, "Revisiting Early Models of the Host-Pathogen Interactions in HIV Infection," Comments Theoretical Biology, Vol. 5, No. 6, 2000, pp. 383411.

[2] M. A. Nowak and R.M. May, "Virus Dynamics: Mathematical Principles of Immunology and Virology," Oxford University Press, New York, 2000.

[3] A. S. Perelson and P. W. Nelson, "Mathematical Analysis of HIV-1 Dynamics in Vivo," SIAM Review, Vol. 41, No. 1, 1999, pp. 3-44.

[4] W.-Y. Tan and H. Wu, "Deterministic and Stochastic Models of AIDS Epidemics and HIV Infections with Intervention," World Scientific, Singapore, 2005.

[5] M. A. Nowak and C. R. M. Bangham, "Population Dynamics of Immune Responses to Persistent Viruses," Science, Vol. 272, No. 5758, 1996, pp. 74-79.

[6] X. Wei, S. K. Ghosh, M. E. Taylor, V. A. Johnson, E. A. Emini, P. Deutsch and J. D. Lifson, "Viral Dynamics in HIV-1 Infection”, Nature, Vol. 273, No. 6510, 1995, pp. 117-122.

[7] X. Xia, "Estimation of HIV/AIDS parameters," Automatica, Vol. 39, No. 11, 2003, pp. 1983-1988.

[8] R. Pattman, M. Snow, P. Handy, K. N. Sankar and B. Elawad, "Oxford Handbook of Genitourinary Medicine, HIV and AIDS," Oxford University Press, USA, 2005.

[9] X. Xia, "Modelling of HIV Infection: Vaccine Readiness, Drug Effectiveness and Therapeutical Failures," Journal of Process Control, Vol. 17, No. 3, 2007, pp. 253-260.

[10] K. R. Fister and S. Lenhart, "Optimizing Chemotherapy in an HIV Model," Journal of Differential Equations, Vol. 1998, No. 32, 1998, pp. 1-12.

[11] K. P. Badakhshan and A. V. Kamyad, "Numerical Solution of Nonlinear Optimal Control Problems Using Nonlinear Programming," Applied Mathematics and Computation, Vol. 187, No. 2, 2007, pp. 1511-1519. 